\title{
Investigation of the Dynamical Response of Methane/Air Counterflow Flames to Inflow Mixture Composition and Flow Field Perturbations
}

\author{
Karin König • Viatcheslav Bykov • Ulrich Maas
}

Received: 3 June 2008 / Accepted: 24 November 2008

(C) Springer Science + Business Media B.V. 2008

\begin{abstract}
This work presents an analysis of the response of laminar, stretched, premixed $\mathrm{CH}_{4}$-air counterflow flames subject to periodical perturbations of the inflow mixture composition and the flow field in the context of ILDM and REDIM. Investigations of the perturbation propagation show, that the perturbation reaches the flame under certain conditions only; changes of the perturbation due to dissipative processes are investigated. Different methods are applied to gain an in-depth view of the influence of the perturbation on the chemical kinetics, namely correlation analyses of species in state space and timescale and element composition analyses. For the timescale analyses, two methods are applied, the ILDM method and a new concept for timescale analysis within the REDIM method. It is shown, that the perturbation does not change the global behaviour of the chemical kinetics and it is suggested to apply REDIMs for a low-dimensional description of perturbed flames.
\end{abstract}

Keywords Perturbed flames • Instationary combustion •

Timescale analysis $\cdot$ ILDM $\cdot$ REDIM

\section{Introduction}

Combustion instabilities are a serious problem in premixed combustion systems like e.g. lean gas turbines and can not only yield a considerable degrading of the efficiency

K. König $(\varangle) \cdot$ V. Bykov · U. Maas

Institut für Technische Thermodynamik,

Universität Karlsruhe (TH), Engelbert-Arnold-Str. 4,

Geb. 10.91, 76131 Karlsruhe, Germany

e-mail: koenig@itt.mach.uni-karlsruhe.de

V. Bykov

e-mail: bykov@itt.mach.uni-karlsruhe.de

U. Maas

e-mail: maas@itt.mach.uni-karlsruhe.de 
of the system, but even a complete breakdown [1]. Reasons for the instabilities are periodical perturbations of the flow field or the reactive mixture equivalence ratio [1], which might be driven by e.g. acoustical properties of the burner or the system of inlet pipes. To understand, how practical combustion systems can be protected against the negative effects of flow field and equivalence ratio oscillations it is necessary to investigate, how the perturbations propagate toward the flame and how they interact with the processes within the flame. Different aspects of perturbations and flame types have therefore already been subject of several publications. A short overview shall be given here. Lean $\mathrm{CH}_{4}$-flames subject to time-dependent perturbations of the strain rate are investigated in [2], focussing on the time delay between perturbation and flame response as well as the amplitude of the flame response due to different perturbation frequencies. Oscillating strain rates are also investigated in [3] by using large activation energy asymptotics to analyse the response behaviour of a counterflow diffusion flame. Fleifil et al. [4] describes the response of a laminar premixed flame stabilized on the rim of a tube subjected to flow oscillations and develops a model which captures the flame surface kinematics. In [1], the behaviour of the heat release of laminar premixed flames during the influence of equivalence ratio oscillations is investigated, finding that the disturbance of flame area and flame speed as well as the heat of reaction control the response of the heat release to the perturbation. A model to predict the stability limits in low $\mathrm{NO}_{\mathrm{x}}$ gas turbines under consideration of the problems arising due to equivalence ratio oscillations is presented in [5]. Darabiha [6] look at $\mathrm{H}_{2}$-air counterflow flames subject to periodical perturbations. Burner-stabilized laminar premixed $\mathrm{CH}_{4}$-air flames with temporal variations of the equivalence ratio are analysed in view of flame temperature and $\mathrm{NO}_{\mathrm{x}}$-emission in [7]. Further aspects of perturbed flames can be found in [8-17]. In the context of reduced mechanisms, [18] presents an investigation of flames under the influence of instationary flow fields using Flamelet Generated Manifolds. More literature on the topic can be found in the references named in the cited publications.

In this work, we present detailed analyses of laminar premixed $\mathrm{CH}_{4}$-air counterflow flames which are subject to perturbations of the flow field or equivalence ratio. We focus on both how the perturbation propagates towards the flame, as well as on the interaction between perturbation and flame. The analyses of the latter are mainly focussed on the chemical kinetics and on developing a concept for describing perturbed flames with reduced mechanisms. There are many approaches to develop reduced chemical kinetics (for an overview see for example [19-21]); we use the two concepts of ILDM (Intrinsic Low Dimensional Manifolds, [22, 23]) and

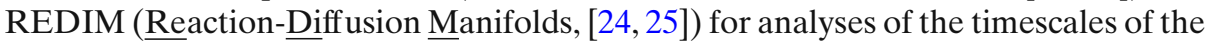
chemical kinetics. Such analyses in the ILDM context have been discussed before in [26] for non-perturbed flames. The REDIM method [24, 25] has not yet been used for analysis purposes.

The paper is organized as follows: Section 2 provides necessary information about the model for the laminar flame calculations and the timescale analyses within the ILDM and REDIM methods. Section 3 discusses the calculation results for both a perturbed inflow composition (Section 3.1) and a perturbed flow field (Section 3.2). First, the perturbation propagation towards the flame is discussed, followed by a discussion of the flame response which is analysed by using state space correlations, timescale analyses and analyses of the element composition. Section 4 summarizes the work and gives concluding remarks. 


\section{Mathematical Background}

\subsection{Laminar flame calculations}

One-dimensional calculations are presented for premixed, stretched $\mathrm{CH}_{4}$-air counterflow flames burning in a laminar flow field. The investigated counterflow configuration is symmetrical, yielding a twin flame in the premixed case (see e.g. [2]). Exploiting the symmetry of both the configuration and the twin flame, the calculation domain can be reduced and thus covers the domain between one burner nozzle and the symmetry plane only. A boundary-layer approximation is used to describe the configuration as a one-dimensional system with the resulting onedimensional conservation equations being well-known [2, 27]. They are solved with a time-accurate instationary approach [28], that has been extended to allow the simulation of counterflow configurations. Adequate time-dependent sinusoidal boundary conditions are applied to simulate the perturbations, who correspond to temporally varying values of the equivalence ratio or flow field. Thus, a inflow mixture composition perturbation is simulated by changing the inflow mixture mass fractions at the cold boundary. The fuel mass fraction $w_{\mathrm{CH}_{4}}(t)$ is given by:

$$
w_{\mathrm{CH}_{4}}(t)=\bar{w}_{\mathrm{CH}_{4}}+\hat{w}_{\mathrm{CH}_{4}} \cdot \sin (2 \pi \mathrm{ft}),
$$

where $\bar{w}_{\mathrm{CH}_{4}}$ is the mean mass fraction value, $\hat{w}_{\mathrm{CH}_{4}}$ the amplitude (in this work we set it to a certain percentage of $\bar{w}_{\mathrm{CH}_{4}}$ ) and $f$ the frequency of the perturbation; $t$ denotes the time. With the fuel mass fraction being changed according to (1), the mass fractions $w_{\mathrm{O}_{2} \text {, air }}(t)$ of $\mathrm{O}_{2}$ and $w_{\mathrm{N}_{2} \text {, air }}(t)$ of $\mathrm{N}_{2}$ can be directly calculated from two natural relations. First, their ratio is constant in air:

$$
\frac{w_{\mathrm{O}_{2}, \text { air }}(t)}{w_{\mathrm{N}_{2}, \text { air }}(t)}=c
$$

with $c$ as the constant. Second, all mass fractions of fuel and oxidizer at the boundary add to 1:

$$
w_{\mathrm{CH}_{4}}(t)+w_{\mathrm{O}_{2}, \text { air }}(t)+w_{\mathrm{N}_{2}, \text { air }}(t)=1 .
$$

Combining these equations yields:

$$
\begin{gathered}
w_{\mathrm{O}_{2}, \text { air }}(t)=\frac{c \cdot\left(1-w_{\mathrm{CH}_{4}}(t)\right)}{1+c}, \\
w_{\mathrm{N}_{2}, \text { air }}(t)=\frac{1-w_{\mathrm{CH}_{4}}(t)}{1+c} .
\end{gathered}
$$

For simulating a perturbed flow field, we impose time-dependent boundary conditions on the strain rate at the cold boundary, which corresponds to varying the mixture outflow velocity. Strain is represented by the tangential pressure gradient $J=-r^{-1} \cdot \partial p / \partial r$ ( $p$ : pressure, $r$ : spatial coordinate), which can also be written as $J=-\rho_{u b} \cdot a^{2}$ with $\rho_{u b}$ as the density of the unburnt mixture density and $a$ as the strain rate. The boundary condition then reads:

$$
J(t)=\bar{J}+\hat{J} \cdot \sin (2 \pi \mathrm{ft})
$$


where again the amplitude $\hat{J}$ is chosen in dependence from the mean value $\bar{J}$. Various perturbation situations can be simulated by changing the three degrees of freedom in (2) and (6), that is the mean perturbation value, amplitude and frequency. It should be furtherly noted, that the calculations are using a detailed reaction mechanism with 34 species and 159 elementary reactions [27] and a detailed transport model with $L e \neq 1$ and non-equal diffusivities. As a result of the calculations we obtain $\boldsymbol{\psi}(\boldsymbol{r})$, which is the vector of thermokinetic state variables (pressure $p$, enthalpy $h$ and species mass fractions $w_{i}(\boldsymbol{r})$, with $i=1, \ldots, n_{s} ; n_{s}$ : number of species) at each spatial location $\boldsymbol{r}$ in the reactive system.

\subsection{Timescale analyses}

An in-depth view onto the interaction between perturbation and flame chemistry can be gained by analysing the timescales of the chemical kinetics. The timescales correspond to the reciprocals of the eigenvalues of the system Jacobian $\boldsymbol{F}_{\boldsymbol{\psi}}(\boldsymbol{\psi})$ (with $\boldsymbol{F}(\boldsymbol{\psi})$ as the chemical source terms), see [27] for details. To analyse the timescales, we use two different concepts. The first concept for a timescale analysis uses the context of ILDM, which means, that the dynamics of the system is said to be governed by chemistry only. The methodology for this kind of analysis has been introduced in [26], where results were discussed for non-perturbed strained and unstrained flames. Results for perturbed flames are presented in this work, though we do not go into detail concerning the numerical method for the timescale analysis, as the approach is thoroughly discussed in [26]. The ILDM method itself and thus also the analysis in its context have a major drawback: As only chemical processes are taken into account, the method using ILDMs of low-dimension provides meaningful results in those flame regions only, where chemistry is fast in comparison to the physical processes and where thus the coupling of chemistry with physical processes such as transport is weak [24]. In regions of slow chemistry-corresponding to the low temperature regime-ILDMs of high dimension would be required to describe the dynamics properly; of course, this is in contrast to the purpose of reduction methods, which aim on finding low-dimensional descriptions for all flame regions. To overcome this drawback, methods have been proposed (see e.g. [24, 29-39]) which include transport processes. The results show, that it is possible to find lowdimensional manifolds even in the regions of slow chemistry. In this work, we focus on the REDIM method [24] to identify low reaction-diffusion manifolds, a method which is currently being developed and improved [24, 25, 40]. Until now, this method has not yet been used for analysis purposes. We present an analysis algorithm in the following, which can be used to determine the necessary REDIM dimension by analysing the detailed flame result. Let us first discuss the important steps to obtain the REDIM equations; afterwards we discuss the analysis algorithm. The notation follows [24], where the REDIM-method was first introduced and discussed. To derive the REDIM-equations, we start from the PDE system describing a reactive flow:

$$
\frac{\partial \boldsymbol{\psi}}{\partial t}=\boldsymbol{F}(\boldsymbol{\psi})-\boldsymbol{v} \cdot \operatorname{grad}(\boldsymbol{\psi})-\frac{1}{\rho} \cdot \operatorname{div}(\boldsymbol{D}(\boldsymbol{\psi}) \cdot \operatorname{grad}(\boldsymbol{\psi}))=\boldsymbol{\Phi}(\boldsymbol{\psi})
$$

$\boldsymbol{v}$ is the flow velocity, $\boldsymbol{D}$ is an $(n \times n)$-dimensional matrix containing the coefficients for energy, pressure and species transport [27] and $\rho$ is the density. Let us now 
assume, that we can find a low-dimensional slow invariant manifold of dimension $m_{s}$ in the $n$-dimensional state space:

$$
M=\left\{\psi=\psi(\theta) ; \theta \varepsilon \mathrm{R}^{\mathrm{m}_{\mathrm{s}}}\right\},
$$

with $\boldsymbol{\theta}$ as an $m_{s}=\left(n-n_{f}\right)$-dimensional vector $\left(n_{f}\right.$ : number of fast processes) representing the local generalized coordinates on the manifold. Note, that $\psi$ is always a function of those generalized coordinates, but for an easier representation we just write $\psi$ in the following. The manifold given by (8) can also be written in an implicit formulation [24], which reads:

$$
\psi_{\theta}^{\perp} \cdot \Phi(\psi)=\mathbf{0}
$$

Therein, $\psi_{\boldsymbol{\theta}}$ is the derivative of $\boldsymbol{\psi}$ with respect to the generalized coordinates. $\boldsymbol{\psi}_{\boldsymbol{\theta}}{ }^{\perp}$ is the orthogonal complement of the tangent subspace of the manifold in a point belonging to the manifold; $\Phi(\boldsymbol{\psi})$ is the right hand side of (7). According to [24], (9) can be re-written by using a projection onto the normal space of the manifold, with the projection being given as

$$
\boldsymbol{P}=\boldsymbol{I}-\psi_{\boldsymbol{\theta}} \cdot \boldsymbol{\psi}_{\boldsymbol{\theta}}{ }^{+}
$$

The resulting equation after projection reads:

$$
\left(\boldsymbol{I}-\boldsymbol{\psi}_{\boldsymbol{\theta}} \cdot \boldsymbol{\psi}_{\boldsymbol{\theta}}{ }^{+}\right) \cdot \boldsymbol{\Phi}(\boldsymbol{\psi})=0,
$$

with the Moore-Penrose pseudo-inverse of $\psi_{\boldsymbol{\theta}}$ [41]:

$$
\boldsymbol{\psi}_{\boldsymbol{\theta}}^{+}=\left(\boldsymbol{\psi}_{\boldsymbol{\theta}}^{T} \cdot \boldsymbol{\psi}_{\boldsymbol{\theta}}\right)^{-1} \cdot \boldsymbol{\psi}_{\boldsymbol{\theta}}^{T}
$$

In [24], an iterative solution procedure is suggested to solve (11), for it is re-written as a parabolic system:

$$
\frac{\partial \boldsymbol{\psi}}{\partial t}=\left(\boldsymbol{I}-\boldsymbol{\psi}_{\boldsymbol{\theta}} \cdot \boldsymbol{\psi}_{\boldsymbol{\theta}}{ }^{+}\right) \cdot \boldsymbol{\Phi}(\boldsymbol{\psi}) .
$$

For a one-dimensional REDIM, it is sure that this equation converges to a fixed point corresponding to the REDIM-solution, it is equivalent to the existence of the stationary system solution. For higher-dimensional cases this question is subject of further research. The starting solution for the iteration procedure is for example given by an extended ILDM (extended ILDMs are discussed in [40]) and the obtained stationary solutions $\boldsymbol{\psi}(\boldsymbol{\theta}, \infty)$ of the equation system (13) yield the manifold $M$ with any converged solution $\psi$ being a state on the manifold.

Let us now discuss, how a already existing flame result can be analysed in the context of REDIM. The analysis process should aim on finding an adequate measure to describe, how good a REDIM of certain dimension approximates the detailed solution in each point of the flame; or, in other words: What is the distance between a point on a REDIM of certain dimension to its corresponding point in the detailed flame solution? Figure 1 illustrates the situation in a sketch of a two-dimensional projection of state space.

The dashed black line corresponds to a REDIM, the circles represent some state in the flame. In the left figure, it can be seen that the angle $\alpha$ between the term $\boldsymbol{\Phi}$, 

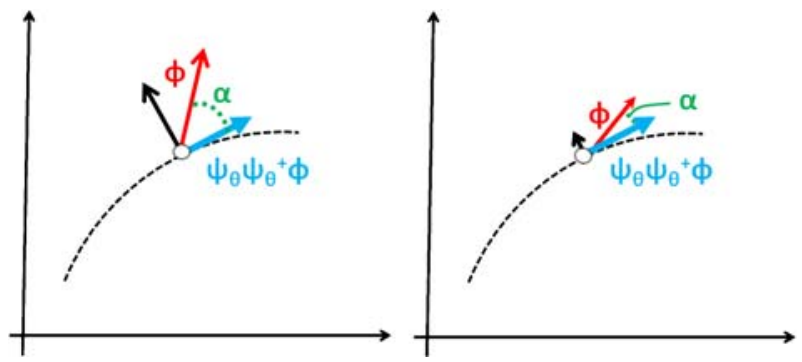

Fig. 1 Schematical plots of a manifold (dashed lines) and the components along the manifold $\psi_{\boldsymbol{\theta}} \psi_{\boldsymbol{\theta}}{ }^{+} \boldsymbol{\Phi}$ and the component driving the movement away from the manifold $\boldsymbol{\Phi}$ with the angle $\alpha$ according to (14). Left figure shows the situation for a point far from the manifold, the right figure for a point near the manifold

which tends to move the dynamics away from the manifold, and the term $\psi_{\boldsymbol{\theta}} \psi_{\boldsymbol{\theta}}^{+} \boldsymbol{\Phi}$, which moves the dynamics along the manifold, is large. On the right side of the figure, the situation is shown for a point near the manifold and it can be well seen, that the angle $\alpha$ is smaller than for the point far away from the manifold. Thus, this angle can be considered an adequate measure to determine the distance between a point in the detailed flame solution and its corresponding point on a local REDIM of given dimension. If the REDIM is a good approximation to the detailed solution, $\alpha$ is small (with $\alpha=0$ if the point on the REDIM and the detailed flame solution point are the same). Of course, this does not give an absolute measure of how good the flame is approximated by the REDIM, but by comparing the angles that result in the single flame points for REDIMs of different dimension, it can well be seen how the quality of the reduced results changes with the REDIM dimension. The equation to calculate the angle $\alpha$ reads:

$$
\cos \alpha=\frac{\boldsymbol{\Phi}^{\mathrm{T}} \cdot\left(\boldsymbol{\psi}_{\boldsymbol{\theta}} \boldsymbol{\psi}_{\boldsymbol{\theta}}^{+}\right) \cdot \boldsymbol{\Phi}}{\|\boldsymbol{\Phi}\| \cdot\left\|\left(\boldsymbol{\psi}_{\boldsymbol{\theta}} \boldsymbol{\psi}_{\boldsymbol{\theta}}^{+}\right) \cdot \boldsymbol{\Phi}\right\|}
$$

Therein, $\boldsymbol{\psi}_{\boldsymbol{\theta}}$ is an $\left(n_{s} \times n\right)$-dimensional matrix containing all terms tangential to the manifold (corresponding to the $n_{s}$ slow processes), $\boldsymbol{\psi}_{\boldsymbol{\theta}}^{+}$is its Moore-Penrose pseudoinverse [41] calculated following (12) (with both matrices being functions of the generalized coordinate $\theta) . \boldsymbol{\psi}_{\boldsymbol{\theta}}$ can easily be calculated, if the REDIM with the dimension of interest is already known. In our case, this REDIM is not known a priori, we just have the detailed flame solution. Thus, we need to find an approximation for $\psi_{\boldsymbol{\theta}}$ that can be calculated based on the local detailed flame solution. A natural choice is to use an ILDM as an approximation; this can be locally calculated (this process is also needed in context of the ILDM analysis, see the description in [26]). Recalling, that the ILDM of a system is given by [27]:

$$
\tilde{\boldsymbol{Z}}_{f}(\boldsymbol{\psi}) \boldsymbol{F}(\boldsymbol{\psi})=0
$$


where $\tilde{\boldsymbol{Z}}_{f}$ is the $\left(n_{f} \times n\right)$-dimensional fast invariant subspace (can be found by a spectral decomposition of the Jacobian $\boldsymbol{F}(\boldsymbol{\psi})$ [27]), we obtain for the terms to be approximated:

$$
\begin{aligned}
& \boldsymbol{\psi}_{\boldsymbol{\theta}}=\left(\tilde{\boldsymbol{Z}}_{f}(\boldsymbol{\psi}) \boldsymbol{F}(\boldsymbol{\psi})\right)_{\boldsymbol{\psi}}^{\perp} \\
& \boldsymbol{\psi}_{\boldsymbol{\theta}}^{\perp}=\left(\tilde{\boldsymbol{Z}}_{f}(\boldsymbol{\psi}) \boldsymbol{F}(\boldsymbol{\psi})\right)_{\boldsymbol{\psi}},
\end{aligned}
$$

where again $\boldsymbol{\psi}$ is a function of the generalized coordinates $\boldsymbol{\theta}$. The derivatives with respect to $\psi$ are in this work computed based on finite differences (analytical differentiation would reduce the computational effort and can also be applied). Using finite differences can yield problems with the (16), as the fast subspaces $\tilde{\boldsymbol{Z}}_{f}$ might be obtained with an arbitrary scaling [42]. Thus, we suggest to use a scalinginvariant formulation as it was also applied in [43]. The scaling invariant equations read:

$$
\begin{aligned}
& \boldsymbol{\psi}_{\boldsymbol{\theta}}=\left(\left(\tilde{\boldsymbol{Z}}_{f}(\boldsymbol{\psi}) \boldsymbol{K}\right)^{-1} \tilde{\boldsymbol{Z}}_{f}(\boldsymbol{\psi}) \boldsymbol{F}(\boldsymbol{\psi})\right)_{\boldsymbol{\psi}}^{\perp} \\
& \boldsymbol{\psi}_{\boldsymbol{\theta}}^{\perp}=\left(\left(\tilde{\boldsymbol{Z}}_{f}(\boldsymbol{\psi}) \boldsymbol{K}\right)^{-1} \tilde{\boldsymbol{Z}}_{f}(\boldsymbol{\psi}) \boldsymbol{F}(\boldsymbol{\psi})\right)_{\boldsymbol{\psi}} .
\end{aligned}
$$

$\boldsymbol{K}$ can be chosen arbitrarily, provided that the resulting product $\tilde{\boldsymbol{Z}}_{f}(\boldsymbol{\psi}) \cdot \boldsymbol{K}$ is regular. A natural choice for $\boldsymbol{K}$ is:

$$
\boldsymbol{K}=\boldsymbol{Z}_{f}(0)=\text { const. } .
$$

Therein, $\boldsymbol{Z}_{f}(0)$ is the right invariant fast subspace in the local point of the detailed flame solution that is subject to the analysis (this matrix is calculated anyway). In the presented equations, $\boldsymbol{\psi}$ includes the chemical kinetics as well as the physical processes. Based on the discussed equations, the angle $\alpha$ can be calculated for different assumed REDIM-dimensions in each point of the detailed flame solution. The results can then be compared, providing a view on how different dimensions of the REDIM affect the distance between a certain point of the detailed flame solution and the corresponding point on a REDIM.

\section{Results and Discussion}

In this section, we present the results of one-dimensional simulations of premixed $\mathrm{CH}_{4}$-air counterflow flames under the influence of perturbations of the inflow mixture composition respectively the flow field. For each of the two perturbation types, we first focus on describing the behaviour of the perturbation on its way towards the flame, then discuss the reaction of the flame in terms of correlation analyses of species in state space. Finally, we present timescale analyses in the ILDM and REDIM context in order to develop a strategy for a reduced description of perturbed flames. All calculations use a detailed $\mathrm{CH}_{4}$ mechanism (34 species, 159 reactions; [27]) and a detailed transport model with unequal Lewis-numbers and unequal diffusivities. The unburnt temperature was assumed to be $298 \mathrm{~K}$, the pressure is kept constant at $p=1$ bar. 
As described in Section 2.1, time-dependent sinusoidal boundary conditions were implemented for the mass fractions at the cold boundary to simulate a perturbation of the inflow mixture composition. The mean values of the species mass fractions were chosen corresponding to a stoichiometric mixture of $\mathrm{CH}_{4}$ and air; the amplitude was set to $10 \%$ of this mean values and frequencies between 20 and $1000 \mathrm{~Hz}$ were investigated. A value of $-0.8 \cdot 10^{6} \mathrm{~N} / \mathrm{m}^{4}$ was assumed for the tangential pressure gradient $J$, which is well below the extinction limit of the stoichiometric flame $\left(J_{\text {ext }} \approx-1.7 \cdot 10^{6} \mathrm{~N} / \mathrm{m}^{4}\right)$.

\subsubsection{Perturbation propagation}

The first questions to be addressed when talking about perturbed flames deal with the perturbation itself, e.g. how does the perturbation reach the flame, is it subject to changes (e.g. in amplitude) on its way to the flame and are there maybe conditions, where the perturbation does not reach the flame? An answer to these questions can be found by analysing the temporal and spatial behaviour of the species $\mathrm{CH}_{4}$, see Fig. 2. In the left figure, specific mole numbers $\left(\phi_{i}=w_{i} / M_{i}\right.$, with $w$ as the mass fraction and $M$ as the molar mass) of $\mathrm{CH}_{4}$ are shown over spatial location and time for different frequencies (top to bottom: $20 \mathrm{~Hz}, 250 \mathrm{~Hz}$, $500 \mathrm{~Hz}, 1000 \mathrm{~Hz}$ ). Two-dimensional slices through these plots for different times are shown on the right. At $20 \mathrm{~Hz}$ (top figures), the perturbation values remain constant over the spatial location and the amplitude of the perturbation is not being changed until the flame zone $(0.005 \ldots 0.007 \mathrm{~m})$ is reached; in other words: At the low frequency, the flame is subject to quasistationary perturbation values, corresponding to different momentary equivalence ratios. For the higher frequencies, different pictures are obtained, showing that the perturbation propagates towards the flame in a wavelike manner. Why we see quasistationary values at low and a wavelike propagation at high frequencies can be explained by the "wavelength", that is imposed on the perturbation at the cold boundary. Calculating this wavelength from the flow field velocity $v$ and the frequency $f$ yields:

$$
\lambda=\frac{v}{f} .
$$

Note, that therein $v$ is chosen to be constant (value taken from the cold boundary), which is an assumption only (of course, the value changes over the flow field). Equation 19 says, that an increasing perturbation frequency yields a decreasing perturbation wavelength. This wavelength can be very long at low frequencies; in our case, the wavelength at a $20 \mathrm{~Hz}$ perturbation frequency is so long, that it clearly exceeds the dimensions of the system and thus, only quasistationary values of the perturbation reach the flame. At higher frequencies, the wavelength is smaller than the system dimensions and thus, a perturbation wave reaches the flame. A further

Fig. 2 Left figures: $\phi_{\mathrm{CH}_{4}}$ plotted versus time and spatial coordinate. Right figures: $\mathrm{CH}_{4}$ plotted versus spatial coordinate at seven different time steps. Inflow mixture composition perturbation with stoichiometric mixture as mean value, amplitude $10 \%$ of the mean value. Frequencies $20 \mathrm{~Hz}, 250 \mathrm{~Hz}$, $500 \mathrm{~Hz}$ and $1000 \mathrm{~Hz}$. Tangential pressure gradient $J=-0.8 \cdot 10^{6} \mathrm{~N} / \mathrm{m}^{4}$ 


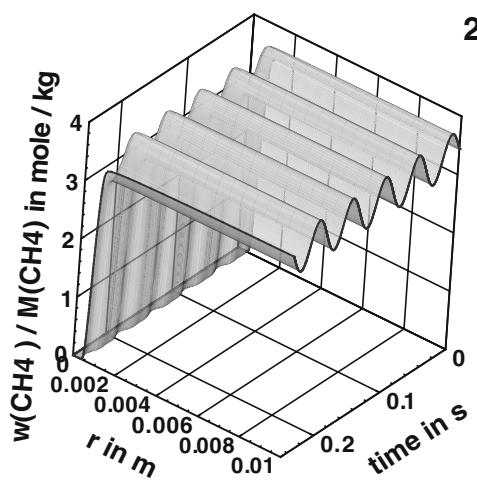

\section{$20 \mathrm{~Hz}$}
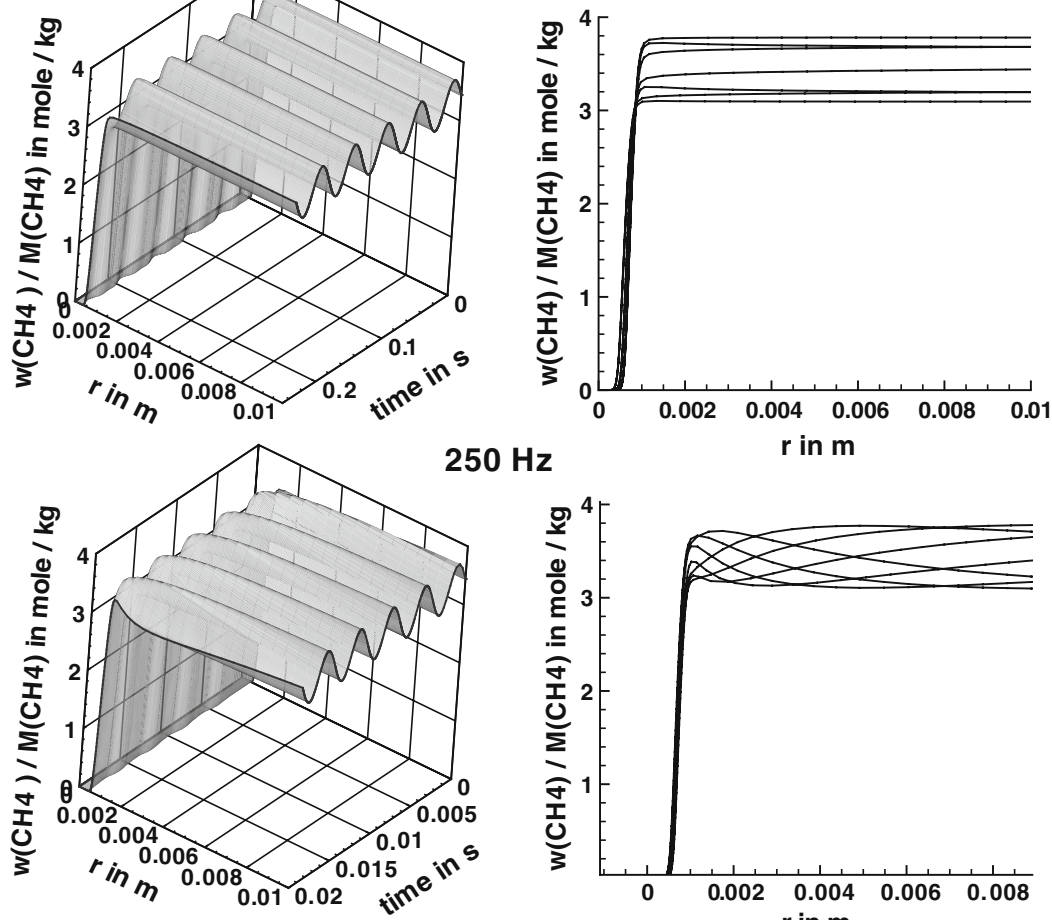

$250 \mathrm{~Hz}$

$r$ in $\mathbf{m}$
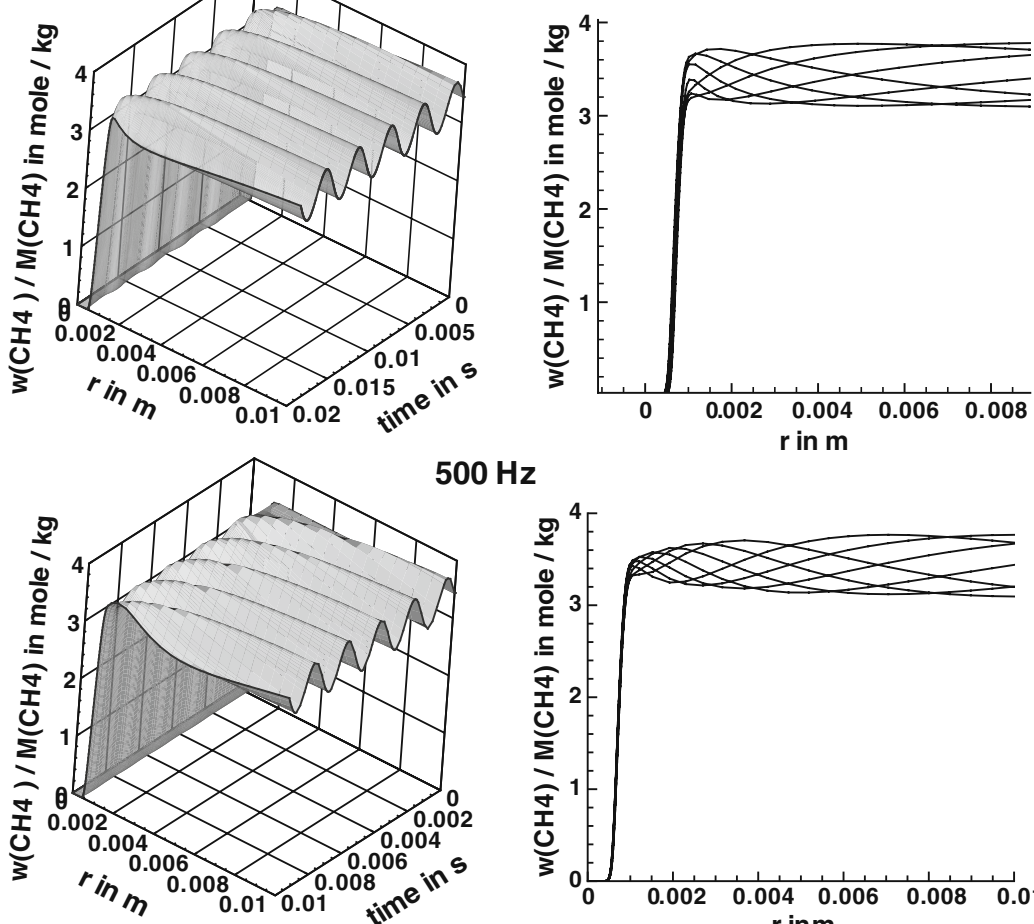

$500 \mathrm{~Hz}$
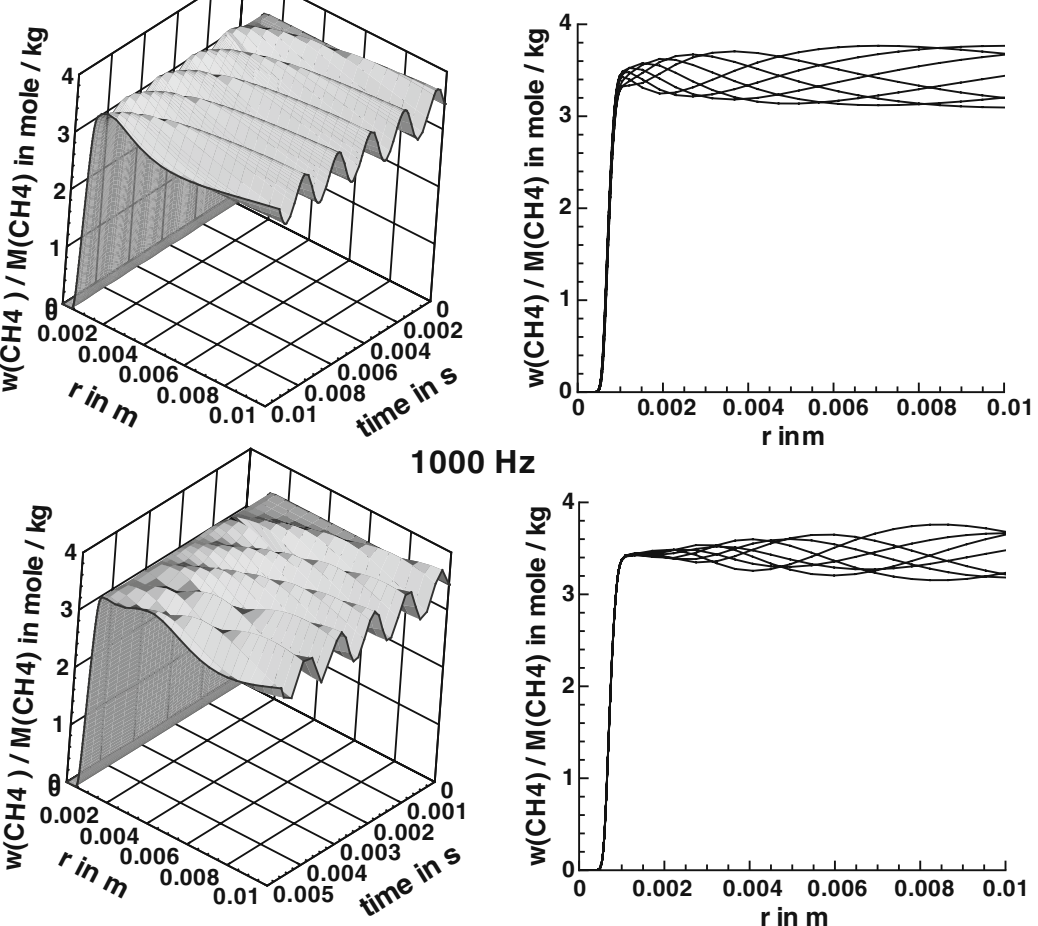
characteristic difference in the perturbation behaviour can be seen from Fig. 2: The higher the frequency, the lower the amplitude that reaches the flame zone. At $1000 \mathrm{~Hz}$, the amplitude of the perturbation is even completely levelled to a mean value. An illustrative explanation for that behaviour can again be found in the context of the wavelength at different frequencies. Equation 19 shows, that an increasing frequency yields a decreasing wavelength. This comes with an increasing curvature of the single wavepeaks and thus causes faster dissipative processes [27], which in turn tend to level the perturbation to a mean value. A simple example can be studied to explain the behaviour mathematically, this is given in the Appendix.

Let us finally summarize the results concerning the behaviour of the perturbation:

- The perturbation propagation towards the flame can be compared to the movement of a wave.

- The wavelength imposed at the cold boundary has to be compared to the system dimensions in order to investigate, whether the perturbation reaches the flame zone.

- Subject to increasing dissipative processes with increasing frequency, the perturbation amplitude decreases on its way to the flame; at high frequencies, the diffusive processes might be so fast, that they have completely levelled the perturbation to the mean value before it reaches the flame.

\subsubsection{State space behaviour}

After investigating the perturbation itself, the influence of the perturbation onto the flame is investigated by analysing correlations of different species in state space. Figure 3 shows two-dimensional projections of the $\left(n_{s}+2\right)$-dimensional state space, with the specific mole numbers of some sample species being shown over the specific mole number of $\mathrm{CO}_{2}$ (which can be considered an adequate measure for the reaction progress, [26]).

The temporal evolution of the species is shown for one full perturbation period at different frequencies $(20 \mathrm{~Hz}$ in black, $250 \mathrm{~Hz}$ in red, $500 \mathrm{~Hz}$ in blue and $1000 \mathrm{~Hz}$ in green). Note, that a detailed transport model (Lewis-number unequal 1 and unequal diffusivities) was used to obtain the results. For $20 \mathrm{~Hz}$, each momentary perturbation value yields a characteristic correlation between the species and a variance between the correlations is obtained due to the varying equivalence ratio. The correlations are in agreement with those that can be found when looking at stationary flames with corresponding equivalence ratios. An explanation for that can be found in the quasistationary values that reach the flame at low frequency without considerably being subject to dissipative processes (see Section 3.1.1). With increasing frequency, a continuous decrease in the variance between species correlations can be found. At $1000 \mathrm{~Hz}$, the correlations are even closely bunched, corresponding to a sharp correlation. This is also consequently following from what was discussed earlier in Section 3.1.1: At high frequencies, the perturbation reaches the flame zone as a mean value only, thus yielding a sharp species correlation. Again, this correlation corresponds to the one obtained from a stationary flame at an adequate equivalence ratio. The correlations being unchanged compared to the stationary case suggest, that the chemical kinetics is not considerably changed by the perturbation. A closer look onto that is possible via timescale analyses, whose results are presented next. 

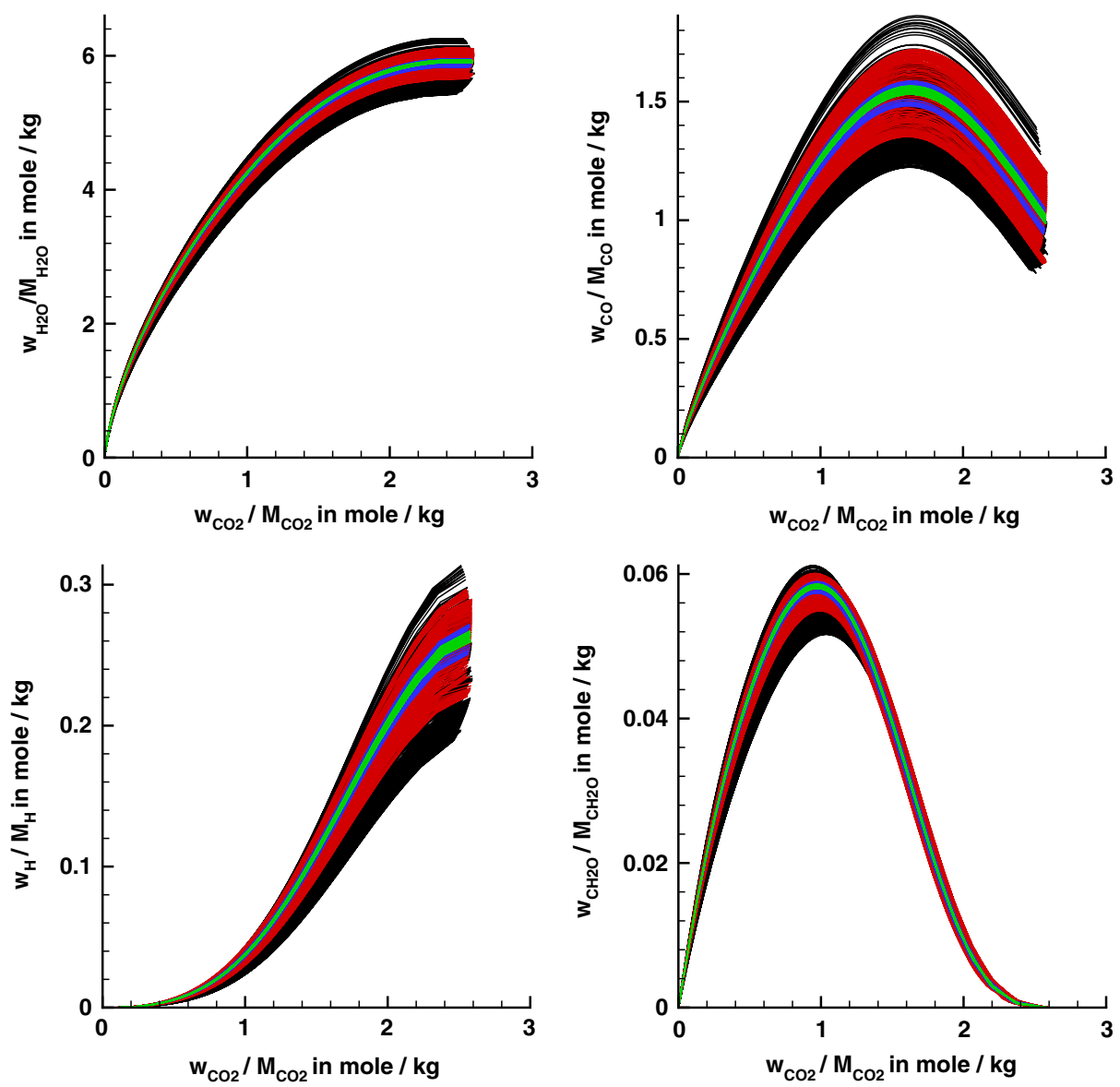

Fig. 3 Projections of the $\left(n_{s}+2\right)$-dimensional state space into two-dimensional subspaces. Shown are different species plotted versus $\mathrm{CO}_{2}$. Results for inflow mixture composition perturbation with stoichiometric mixture as mean value, amplitude $10 \%$ of the mean value, frequencies $20 \mathrm{~Hz}$ (black), $250 \mathrm{~Hz}($ red $), 500 \mathrm{~Hz}$ (blue) and $1000 \mathrm{~Hz}$ (green). Tangential pressure gradient $J=-0.8 \cdot 10^{6} \mathrm{~N} / \mathrm{m}^{4}$

\subsubsection{Timescale analyses}

Timescale analyses in both the ILDM and REDIM context (see Section 2.2) are now presented to gain a in-depth view onto the chemical kinetics in the perturbed flames. In order to simplify the analyses, the knowledge about the behaviour of the perturbation from Section 3.1.1 can be exploited:

- Analyses of stationary flames at adequate values of the inflow mixture compositions are sufficient, if only quasistationary values of the perturbation reach the flame; this is at low (wavelength exceeding system dimensions) and at very high frequencies (perturbation levelled by diffusive processes).

- Analyses of instationary flames are only necessary in between the two extremes described before. 
Therefore, we analyse stationary flames at the equivalence ratios $\Phi=1.0,1.1$ and 0.9 (corresponding to minimum, mean and maximum perturbation value) and a perturbed flame at $500 \mathrm{~Hz}$ (see the discussion in Section 3.1.1). It should be added, that the calculations of the stationary flames use the same boundary conditions than the perturbed ones with the perturbed mass fractions being replaced by fixed values.

First, we discuss the stationary flame analyses. Strained and unstrained unperturbed flames have already been discussed in the ILDM context in [26], but not at different equivalence ratios. Figure 4 shows the results of the analyses for three equivalence ratios, with solid lines being used for $\Phi=1.0$, dotted ones for $\Phi=0.9$ and dashed ones for $\Phi=1.1$. The left figure shows the 15 largest eigenvalues (the system has 36 eigenvalues) versus $\phi_{\mathrm{CO}_{2}}$ and the right figure the corresponding number of relaxed modes calculated on basis of a local error $\leq 5 \%$, also plotted versus $\phi_{\mathrm{CO}_{2}}$. For all equivalence ratios, 6 of the shown eigenvalues equal 0 , corresponding to the conserved quantities in the system (enthalpy, pressure and elements). Only one of the eigenvalues has a positive real part, while all others are negative and can therefore be identified as belonging to relaxation processes. For $\phi_{\mathrm{CO}_{2}}$ values between 0 and $1.0 \mathrm{~mole} / \mathrm{kg}$, all shown eigenvalues are closely bunched. Correspondingly, the number of relaxed modes obtained from the ILDM analysis is small (between 10 and 15) at all equivalence ratios. A low number of relaxed modes corresponds to an ILDM of high dimension that is needed to describe the system within the required accuracy (difference between detailed and reduced solution $<5 \%$ ). An explanation for that can be found in the domain of slow chemistry, were there is no clear separation between slow and fast eigenvalues (as can be seen from the top part of the figure) $[22,23]$. With increasing $\phi_{\mathrm{CO}_{2}}$ values, especially from $\phi_{\mathrm{CO}_{2}}=2.0 \mathrm{~mole} / \mathrm{kg}$ on, the separation becomes clearer and thus, the number of relaxed modes increases. For the maximum $\phi_{\mathrm{CO}_{2}}$ the number of relaxed modes is 28 , corresponding to a two-dimensional ILDM that would be sufficient to describe the system within the given accuracy. It is important, that the described behaviour of the eigenvalues and relaxed modes does not qualitatively differ for the three equivalence ratios. At all
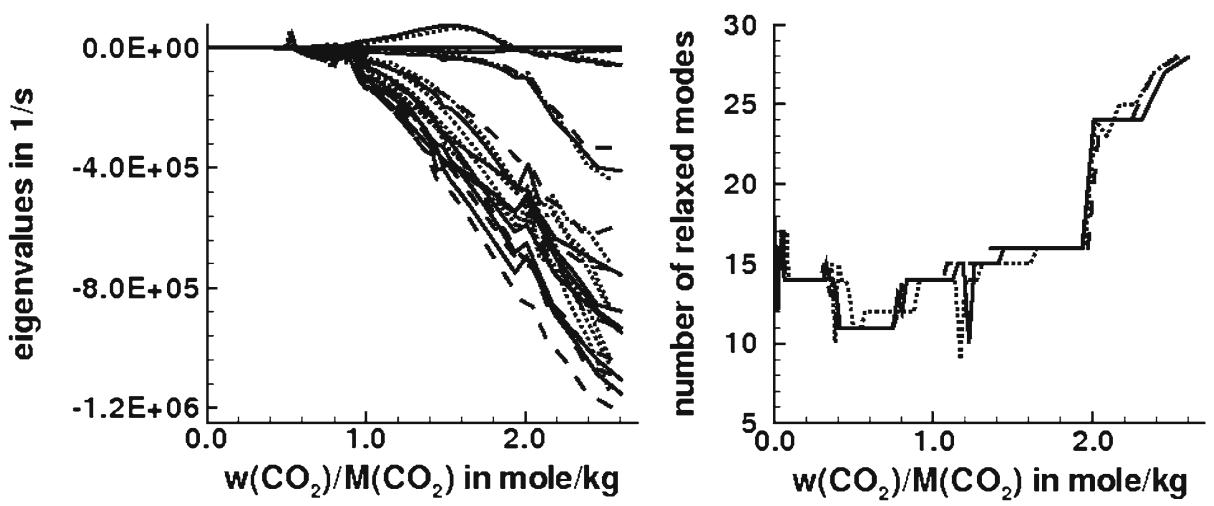

Fig. 4 Eigenvalue spectra (left figure) and number of relaxed modes (right figure) plotted versus $\phi_{\mathrm{CO}_{2}}$ for stationary flames with stoichiometric mixture (solid lines) and equivalence ratios 0.9 (dotted lines) and 1.1 (dashed lines). The number of relaxed modes has been calculated with an ILDM-based analysis based on a local error between reduced and detailed solution of $<5 \%$. Tangential pressure gradient $J=-0.8 \cdot 10^{6} \mathrm{~N} / \mathrm{m}^{4}$ 
equivalence ratios the locally needed ILDM dimension is similar. It can thus be stated, that an ensemble of unperturbed strained flames at different equivalence ratios, corresponding to a perturbed flame at a low frequency, can be described by the same ILDM dimension. Of course, additional dimensions or coordinates have to be included in the ILDMs to represent the changing equivalence ratio and the resulting different element compositions. For a discussion of the element composition issue see Section 3.1.4. Furthermore, it has to be investigated whether the behaviour of the eigenvalues and the number of relaxed modes changes, when the flame is perturbed. We analyse the perturbed flame with a frequency of $500 \mathrm{~Hz}$; Fig. 5 shows the results for different times corresponding to different momentary inflow compositions (at $8.5 \cdot 10^{-3} \mathrm{~s}, 9.5 \cdot 10^{-3} \mathrm{~s}$ and $1.0 \cdot 10^{-2} \mathrm{~s}$; all times within the fifth perturbation period, when transient effects have vanished). Again, the top figure shows the eigenvalues and the bottom figure the number of relaxed modes, both being plotted versus $\phi_{\mathrm{CH}_{4}}$. It can well be seen, that the qualitative behaviour is the same that could be obtained from analyses of stationary flames: There are no considerable differences in the qualitative behaviour between the different times. The eigenvalues show even smaller differences for the different times respectively equivalence ratios than they did for the stationary flames. This can be attributed to the perturbation loosing amplitude due to dissipative processes (see Section 3.1.1). The number of relaxed modes does not change its global behaviour, too. No additional slow timescales are added by the perturbation (but note, that the number of slow timescales is relatively high anyway) and it is possible to use the same dimension to describe the dynamics of the system at all timesteps. Summarizing what was found so far from the timescale analyses, it can be said that:

- The global behaviour of the eigenvalues, especially the separation between the timescales is not changed in the perturbed case compared to the unperturbed ones.

- No additional slow timescales are obtained in the perturbed case.
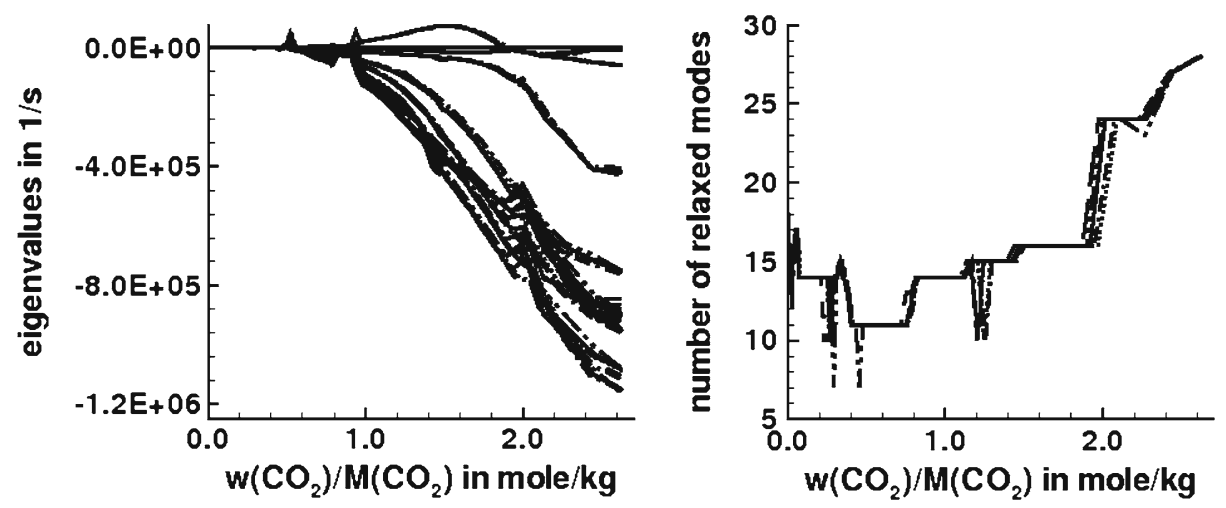

Fig. 5 Eigenvalue spectra plotted versus $\mathrm{CO}_{2}$. Results for inflow mixture composition perturbation with stoichiometric mixture as mean value, amplitude $10 \%$ of the mean value, frequency $500 \mathrm{~Hz}$ at six different time steps. The number of relaxed modes has been calculated with an ILDM-based analysis based on a local error between reduced and detailed solution of $<5 \%$. Tangential pressure gradient $J=-0.8 \cdot 10^{6} \mathrm{~N} / \mathrm{m}^{4}$ 
- The number of relaxed modes, which corresponds to the local number of processes that can be decoupled from the system, is globally not changed by the perturbation.

- The overall number of relaxed modes is relatively low (corresponding to a high ILDM dimension that is needed) in wide regions of the flames.

The last mentioned issue is well-known, as the ILDM-method does not account for diffusive processes [24]. As described before in Section 2.2, this problem can be dealt with by using methods like REDIM [24], that consider diffusion for constructing a low-dimensional manifold. In order to analyse, which REDIM dimension would be sufficient to describe the flames, we now use the analysis algorithm proposed in Section 2.2. The analysis is done for unperturbed flames at different equivalence ratios only; this is a simplification that is justified by the results from the ILDM analyses, that did not show a change in the behaviour of the chemical kinetics in the perturbed case. The analysis is done by calculating the angle $\alpha$ in each point of the detailed flame calculation for different assumed REDIM dimensions. In Fig. 6, the angle is being plotted versus $\phi_{\mathrm{CO}_{2}}$ for the stationary flames at equivalence ratios $\Phi=1.0$ (solid lines), $\Phi=0.9$ (dotted lines) and $\Phi=1.1$ (dashed lines). REDIM dimensions of one (black), two (blue), three (red) and ten (green) were assumed. For $\phi_{\mathrm{CO}_{2} \text {, max }}$ and $\phi_{\mathrm{CO}_{2}}=2.0 \mathrm{~mole} / \mathrm{kg}$, a gap can be seen between the dimensions one and two and between dimensions two and three. That means, that a two-dimensional description would yield a considerably better result than a one-dimensional description, as well as a three-dimensional description would further increase the quality of the result. Around $\phi_{\mathrm{CO}_{2}}=1.5 \mathrm{~mole} / \mathrm{kg}$, the gap is big between dimensions one and two, but not between two and three, thus, a dimension increase to three is not essential in this region. For values smaller than $\phi_{\mathrm{CO}_{2}}=1.5 \mathrm{~mole} / \mathrm{kg}$ the gap can mainly seen between dimensions two and three. For dimension three, the angle is already very small, what can be interpreted in the way, that dimension three should provide very good results in comparison to the detailed solution. This is in good agreement with results found in [24], where even calculations using REDIMs of dimension two provided very good results in comparison to the detailed solution.

Fig. 6 Angle $\alpha$, see (14), plotted over $\phi_{\mathrm{CO}_{2}}$ calculated for stationary flames with stoichiometric mixture (solid lines) and equivalence ratios 0.9 (dotted lines) and 1.1 (dashed lines). Assumed REDIM dimensions 1 (black lines), 2 (blue lines), 3 (red lines) and 10 (green lines). Tangential pressure gradient $J=-0.8 \cdot 10^{6} \mathrm{~N} / \mathrm{m}^{4}$

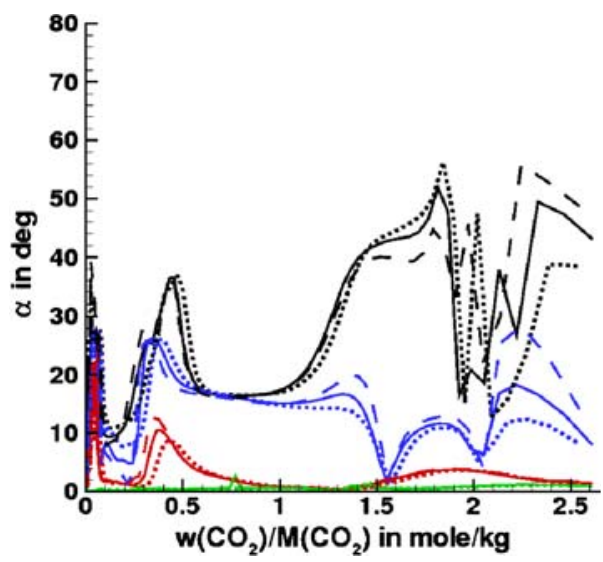


To get an idea about how the angle changes for even higher dimensions, it was also calculated for an assumed REDIM dimension of ten (green line). It can be obtained, that the angle decreases further, with the value being close to zero; this means, that some points in the flame are nearly elements of the ten-dimensional REDIM. Even though this higher dimension is expected to provide better reduced results (probably mostly for minor species), other aspects have to be considered when choosing a REDIM dimension for reduced calculations, that is the computational effort for the calculation of the manifold, the needed storage capacity for the table and the reached level of reduction.

\subsubsection{Element composition}

Dealing with the needed dimension of an ILDM or REDIM does not only require to look at the number of slow and fast processes, as it was done in Section 3.1.3, but it must also be discussed, what happens to the element composition. ILDM or REDIM requires an additional coordinate, when the element composition is changing; e.g. in a non-premixed flame, where the mixture fraction has to be included in ILDMor REDIM-tables as a coordinate. It is therefore of interest to investigate, which correlations govern the element composition in perturbed flames and whether they can be compared to the element composition correlations in unperturbed flames.

Describing the element composition in an unperturbed, premixed $\mathrm{CH}_{4}$-air flame requires the knowledge of four different conditions, with three of them being given directly:

- The sum of all element mass fractions equals 1.

- The ratio between $\mathrm{C}$ and $\mathrm{H}$ in $\mathrm{CH}_{4}$ is constant.

- The ratio between $\mathrm{O}$ and $\mathrm{N}$ in air is constant.

The fourth correlation is e.g. given by the equivalence ratio of the $\mathrm{CH}_{4}$-air mixture. If an ensemble of such stationary flames at different equivalence ratios should be described, additional two correlations are required. The question to be answered is, whether these two correlations are also sufficient to describe a perturbed flame or whether the perturbation introduces further correlations that are needed to properly describe the element composition. Therefore, the behaviour of the element composition in a perturbed flame at $500 \mathrm{~Hz}$ is compared to that in stationary flames of different equivalence ratios. We start from stationary solutions of 44 unperturbed flames with equivalence ratios between $\phi=0.88$ to $\phi=1.1$ (covering the range of equivalence ratios caused by the perturbation). These are used to build up a table which can be used for the comparison with the perturbed flame. In single points on the trajectories of the perturbed flame, we then calculate the orthogonal distance between the flame point and the plane spanned by the next three neighbours of the point from the table. Results can be seen in Fig. 7. The two-dimensional correlation between the stationary flames at different equivalence ratios is shown as grey area. The solutions of the $500 \mathrm{~Hz}$ perturbed flames at different times are shown as coloured symbols. Therein, the colours represent the orthogonal distance between the perturbed flame point and the plane spanned by its next three neighbours in the stationary flames table. The distances are very small, which indicates, that the element compositions in the perturbed flame are-in good approximation-points of the plane spanned by element compositions of unperturbed flames. Summarizing, it 
Fig. 7 Table of element compositions of stationary flames, $\phi=0.88 \ldots 1.1$, shown as grey area. Element compositions for perturbed flame with $500 \mathrm{~Hz}$ and 10\% amplitude at different time steps (marked with different symbols). Distance of solution of perturbed flame to stationary solutions used as contour colour, see legend. Tangential pressure gradient $J=-0.8 \cdot 10^{6} \mathrm{~N} / \mathrm{m}^{4}$

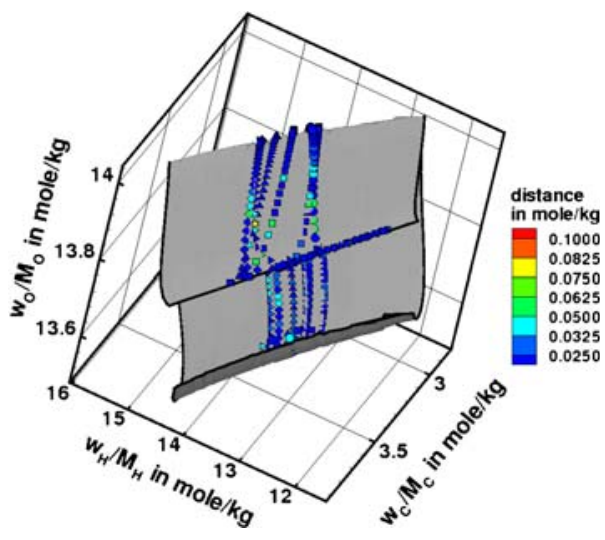

can be said that the correlations between the element compositions in the perturbed flames are-in good approximation-the same as in an ensemble of corresponding stationary flames. No additional correlations are added by the perturbation. Therefore, the governing correlations for the element composition can be obtained from a lookup table of corresponding unperturbed flames. The tables might also be used to estimate the influence of the perturbation on the flame when used for comparisons between perturbed results and the table.

\subsection{Flow field perturbation}

Flow field perturbations were imposed on stoichiometric premixed $\mathrm{CH}_{4}$-air counterflow-flames by implementing adequate time-dependent boundary conditions for $J$ on the cold boundary (see Section 2.1). In (6), the mean perturbation value was chosen to be $\bar{J}=-0.8 \cdot 10^{6} \mathrm{~N} / \mathrm{m}^{4}$ and the amplitude $\hat{J}=0.50 \cdot \bar{J}$, resulting in minimum / maximum perturbation values of $-0.4 \cdot 10^{6} \mathrm{~N} / \mathrm{m}^{4}$ respectively $-1.2 \cdot 10^{6} \mathrm{~N} / \mathrm{m}^{4}$ (extinction limit $J_{\text {ext }} \approx-1.7 \cdot 10^{6} \mathrm{~N} / \mathrm{m}^{4}$ ). Frequencies have been varied between 20 and $1000 \mathrm{~Hz}$.

\subsubsection{Perturbation propagation}

To illustrate the propagation of the perturbation, the flow field velocity is analysed over the spatial coordinate at different time steps and frequencies, see Fig. 8. Like before for the perturbed inflow mixture composition, the propagation of the perturbation at high frequencies corresponds to a wavelike movement. At low frequencies, the perturbation has again a very long wavelength which exceeds the dimensions of the system, resulting in quasistationary perturbation values in the flame region. At all frequencies, the amplitude reaching the flame zone is very small and thus the representation in Fig. 8 is not sufficient to gain a detailed view onto what happens to the amplitude on its way to the flame. Therefore, we choose a different representation with the maximum change of the flow field velocity (maximum minus minimum value, corresponding to the double amplitude) being plotted over the spatial location for one full perturbation period, see Fig. 9. To make a comparison 

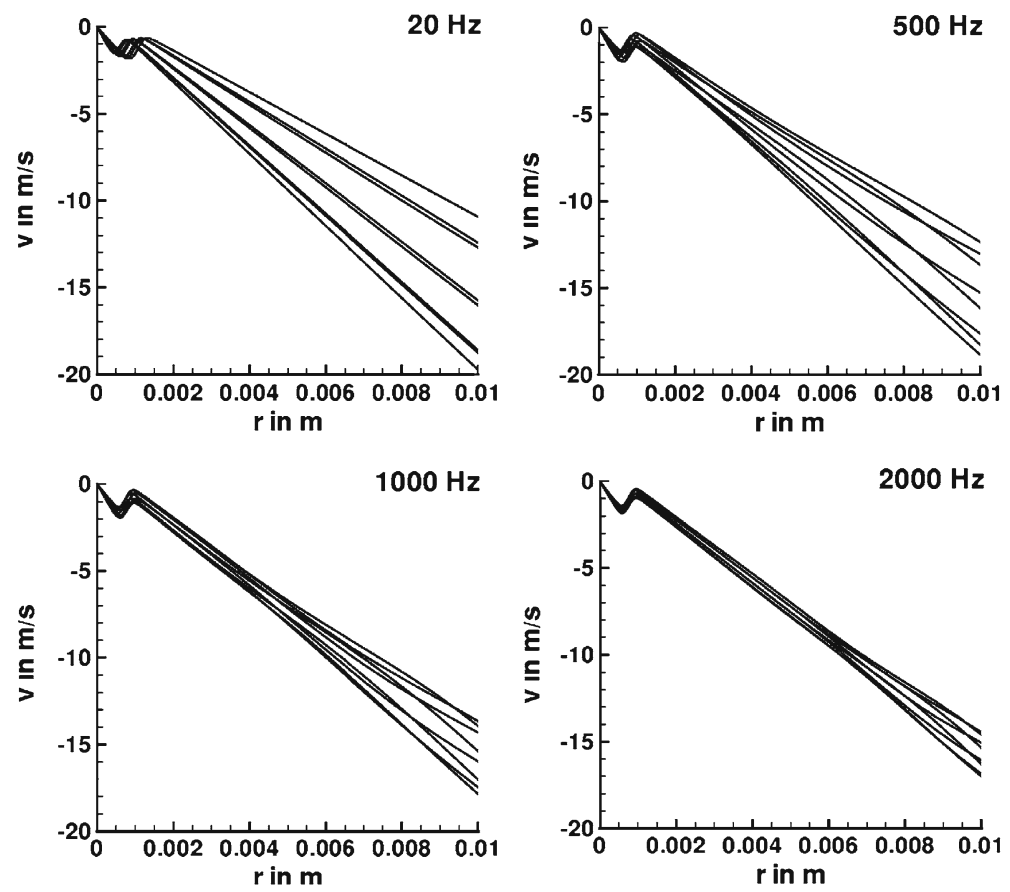

Fig. 8 Flow field velocity versus spatial coordinate for different frequencies (top figures: left $20 \mathrm{~Hz}$, right $500 \mathrm{~Hz}$; bottom figures: left $1000 \mathrm{~Hz}$, right $2000 \mathrm{~Hz}$ ). Stoichiometric $\mathrm{CH}_{4}$-air flame with a perturbation of the tangential pressure gradient; $\bar{J}=-0.8 \cdot 10^{6} \mathrm{~N} / \mathrm{m}^{4}, \hat{J}=0.50 \cdot \overline{\mathbf{J}}$

possible between the different frequencies, the value of the flow field velocity was scaled with its respective cold boundary value. In this figure, a decrease in the change of $v$ can be seen with increasing frequency. This corresponds to the observation made for the perturbed inflow mixture composition. But there is a difference, too: Whereas the inflow mixture perturbation reached the flame without a considerable loss of amplitude at low frequencies, the change of $v$ is subject to a noticeable decrease even

Fig. 9 Maximum change of flow field velocity over spatial coordinate for different frequencies. Values normed to cold boundary value for each frequency. Stoichiometric $\mathrm{CH}_{4}$-air flame with a perturbation of the tangential pressure gradient;

$\bar{J}=-0.8 \cdot 10^{6} \mathrm{~N} / \mathrm{m}^{4}$, $\hat{J}=0.50 \cdot \overline{\mathbf{J}}$

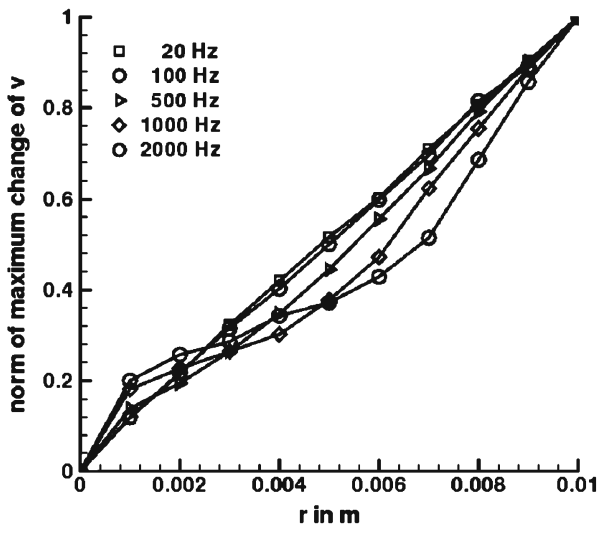


at this low frequency. At $20 \mathrm{~Hz}$, this decrease is nearly linear. Different phenomena can be explained by the so far discussed behaviour of the perturbation:

- In [2], it was shown that the amplitude of the flame response decreases with increasing amplitude. Together with the described behaviour of the perturbation, this can be attributed to the perturbation being dissipated on its way to the flame: The flame is not subject to the maximum and minimum perturbation values at the boundary, but to reduced values only, which yield smaller flame reactions.

- Flames which are perturbed with high frequencies are able to sustain maximum perturbation values beyond their extinction limit. This can also be explained by the dissipation of the maximum perturbation values before the flame zone.

- The delay between perturbation and flame response increases with increasing frequency [2]. Reason for that can be found in the wavelike movement of the perturbation, which yields that the momentary boundary values are equal to the momentary values at the flame. Thus, the reaction of the flame is not instantaneous and this delay increases with increasing frequency as the perturbation is more and more dissipated before reaching the flame.

With this knowledge about the behaviour of the perturbation we can now switch to analyses of its impacts on the flame. As the state space analyses do not bring any new observations than those found for the perturbed inflow mixture composition (Section 3.1.2), we do not discuss them here.

\subsubsection{Timescale analyses}

It was described before, that only small changes of $v$ reach the flame at all frequencies. Therefore it is sufficient to analyse unperturbed stationary flames at different flow field velocities. Such variations of the flow field velocity correspond to calculating flames at different strain rates (tangential pressure gradients). In the ILDM-context, such stationary flames with different strain rates were investigated in [26] and are therefore not discussed here. The results there showed, that ILDMs can be used to describe strained flames in the entire flame domain, with the number of relaxed modes being high (between 29 and 25) for $\phi_{\mathrm{CO}_{2}}>2.0 \mathrm{~mole} / \mathrm{kg}$ and the number being not smaller than 12 for smaller $\phi_{\mathrm{CO}_{2}}$ values. The global behaviour of the eigenvalues, which is shown at the top part of the figure, does not change for the different strain rates. The eigenvalues are closely bunched until $\phi_{\mathrm{CO}_{2}} \approx 1.0 \mathrm{~mole} / \mathrm{kg}$ and are then evolving with characteristic gaps, which are important for the decoupling in the ILDM/REDIM context. Correspondingly, the number of relaxed modes in the bottom figure is small in those flame regions, where the separation of the eigenvalues is not clear. It increases with increasing gaps between the eigenvalues, for example for $\phi_{\mathrm{CO}_{2}} \approx 1.9 \mathrm{~mole} / \mathrm{kg}$. Due to the overall low number of relaxed modes, it is again strongly recommended to use REDIMs for the reduced description. Concerning a fixed dimension of the REDIM that can be used in the whole domain, we again look at the angle $\alpha$ according to (14) for different REDIM dimensions, see Fig. 10. The angle was calculated for different strain rates corresponding to the mean, minimum and maximum perturbation value (solid lines: $J=-0.8 \cdot 10^{6} \mathrm{~N} / \mathrm{m}^{4}$, dotted lines: $J=-0.4 \cdot 10^{6} \mathrm{~N} / \mathrm{m}^{4}$, dashed lines: $J=-1.2 \cdot 10^{6} \mathrm{~N} / \mathrm{m}^{4}$ ). Dimensions one (black lines), two (blue lines), three (red lines) and ten (green lines) were considered. First, the qualitative behaviour as well as the magnitude of the angle at a certain dimension is not changed by the different values of strain rate. Thus, a table of the same 
Fig. 10 Angle $\alpha$, see (14), plotted over $\phi_{\mathrm{CO}_{2}}$ calculated for stationary flames with $J=-0.8 \cdot 10^{6} \mathrm{~N} / \mathrm{m}^{4}$ (solid lines), $J=-0.4 \cdot 10^{6} \mathrm{~N} / \mathrm{m}^{4}$ (dotted lines) and $J=-1.2 \cdot 10^{6} \mathrm{~N} / \mathrm{m}^{4}$ (dashed lines). Assumed REDIM dimensions 1 (black lines), 2 (blue lines), 3 (red lines) and 10 (green lines). Stoichiometric mixture

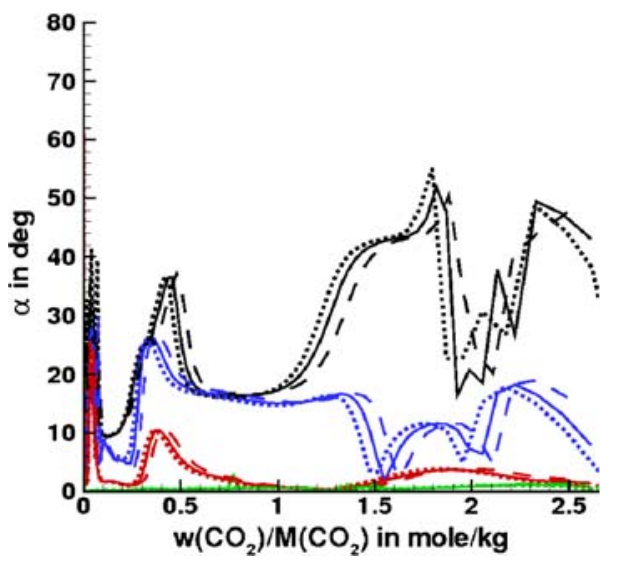

dimension can describe all strain rates at the same level of accuracy. Concerning the different dimensions, a gap can be obtained between dimensions one and two respectively three, with the angle being small at dimension three. As before for the different equivalence ratios, it can therefore be stated, that REDIM dimensions two and three should be considered for reduced calculations. Again, dimension ten shows a further decrease of the angle. However, this further decrease (corresponding to a further improvement of the reduced result) does not seem to justify the higher effort that is connected to tables of such high dimension.

\subsubsection{Element composition}

A detailed transport model with unequal diffusivities and $L e \neq 1$ was used for the calculations. This means, that a change of the element composition can occur due to differential diff usion even though the stoichiometry of the flame was not changed and the element composition has to be analysed in order to find correlations to describe the behaviour of the element composition. The same analysis strategy was applied that we already used in Section 3.1.4. The lookup-table of different stationary flames was set up for flames at $\phi=1.0$ with different strain values and the perturbed flames were compared to that table. The results correspond to the ones found in Section 3.1.4, with the behaviour of the element composition in perturbed flames being directly comparable to the behaviour in the stationary flames. Again, no correlations vanish due to the perturbation.

\section{Summary and Conclusions}

Laminar premixed $\mathrm{CH}_{4}$-air counterflow flames were investigated under conditions of a perturbed inflow mixture composition respectively a perturbed flow field using one-dimensional simulations with adequate time-dependent boundary conditions. For both perturbations it could be found, that the propagation of the perturbation towards the flame corresponds to a wavelike movement and that the perturbation amplitude is subject to dissipative processes dependent on the frequency. The amplitude reaching the flame decreases with increased frequency and might even 
be completely levelled to a mean value before the flame zone. State space analyses of species and timescale analyses showed, that the perturbations do not qualitatively influence the behaviour of the chemical kinetics. The local number of relaxed modes in the flame was identified using an ILDM-based timescale analysis. Results show, that the number of relaxed modes is globally not influenced by the perturbations and that it is possible to decouple a considerable number of processes from the system in the whole domain of interest. In some regions of the flame, the ILDM concept does not provide a sufficient reduction of the system, thus it is suggested to use REDIMs. These newly proposed manifolds (see e.g. [24]) account for diffusion processes and are thus able to provide a low-dimensional description over the whole flame. In this work, we presented a new analysis algorithm in the context of REDIM and applied it in the discussed context of perturbed flames. It showed, that REDIMs of dimensions two and three provide reduced results that are close to the detailed flame solution throughout the whole domain of the flames. As a changing element composition has to be included in ILDM/REDIM-tables, we compared the behaviour of the element composition in perturbed flames to that in corresponding unperturbed flames; it was shown, that the correlations between the element compositions are not changed by the two types of perturbation. Therefore, a lookup-table consisting of stationary flames can be used to describe the two-dimensional correlations of element compositions in perturbed flames.

Acknowledgements The authors express their thanks to the Deutsche Forschungsgemeinschaft, who supported this work within the framework of the Collaborative Research Center 606 and CIVB (Combustion Induced Vortex Breakdown).

\section{Appendix}

Example for frequency dependent loss of amplitude

Consider a parabolic equation and its boundary conditions including the perturbation of the unburnt state. Note, that we look at the system from a Lagrangian point of view and do therefore not include convection; this is justified, as convection processes do not change a manifold [22,23]:

$$
\begin{gathered}
\psi_{t}=k \psi_{x x} \\
t>0,0<x<1 \\
\psi(0, t)=\Lambda \sin (\omega \mathrm{t}), \psi(1, \mathrm{t})=0, \psi(\mathrm{x}, 0)=0 .
\end{gathered}
$$

$x$ is given as the coordinate of each point scaled with the system length, $k$ are the diffusion coefficients, also scaled with the system length. Equation 20 is transformed into a homogeneous form with respect to the boundary conditions. Then, we apply the methods of Fourier expansion and separation of variables, that means, we are looking for:

$$
\begin{aligned}
& v(x, t)=\psi(x, t)-(1-x) \Lambda \sin (\omega \mathrm{t}) \\
& v(0, t)=\psi(0, t)-\Lambda \sin (\omega \mathrm{t}) \equiv 0 \\
& v(1, t)=\psi(1, t) \equiv 0, v(x, 0)=\psi(x, 0) \equiv 0 .
\end{aligned}
$$


Simple calculations yield a non-homogeneous system for $v(x, t)$ with homogeneous boundary conditions:

$$
\begin{gathered}
v_{t}=k v_{x x}-(1-x) \Lambda \omega \cos (\omega \mathrm{t}), \mathrm{t}>0,0<\mathrm{x}<1 \\
v(0, t)=0, v(1, t)=0, v(x, 0)=0 .
\end{gathered}
$$

A standard solution for this system can be found through separation of variables and Fourier series as

$$
v(x, t)=\sum_{n=1}^{\infty} \int_{0}^{t} e^{k \lambda_{n}(t-s)} F_{n}(s) d s \sin (\mathrm{n} \pi \mathrm{x})
$$

with

$$
\begin{gathered}
\lambda_{n}=-(n \pi)^{2} \\
F_{n}(t)=2 \int_{0}^{1} F(x, t) \sin (\mathrm{n} \pi \mathrm{x}) \mathrm{dx} .
\end{gathered}
$$

Here, $F(x, t)$ denotes the additional nonhomogeneous term $F(x, t)=-(1-$ $x) \Lambda \omega \cos (\omega t)$ in (22) and $\lambda_{n}$ is the eigenvalue for the $\mathrm{n}$-th mode. By using the sine Fourier series $(1-x)=\frac{2}{\pi} \sum_{n=1}^{\infty} \frac{n \pi x}{n}$, we can write the final solution in form of the series:

$$
\begin{aligned}
\psi(x, t) & =v(x, t)+(1-x) \Lambda \sin (\omega \mathrm{t}) \\
& =\sum_{n=1}^{\infty}\left\{\int_{0}^{t} e^{k \lambda_{n}(t-s)} F_{n}(s) d s+\frac{2 \Lambda \sin (\omega \mathrm{t})}{\pi n}\right\} \sin (\mathrm{n} \pi \mathrm{x}) .
\end{aligned}
$$

To see, how different frequencies $\omega$ affect the solution, we have to analyse the term $a_{n}(t)=\{\ldots\}$, which is defining the amplitudes of the Fourier expansion. Simplification and integration permits to write it in an explicit form:

$$
\begin{aligned}
F_{n}(t)=-\frac{2 \Lambda \omega \cos (\omega \mathrm{t})}{\pi n} & \Rightarrow a_{n}=\frac{2 \Lambda}{\pi n}\left(-\int_{0}^{t} e^{k \lambda_{n}(t-s)} \omega \cos (\omega \mathrm{t}) \mathrm{ds}+\sin (\omega \mathrm{t})\right) \\
& \Rightarrow a_{n}=\frac{2 \Lambda k \lambda_{n}}{\pi n}\left(\frac{-\omega e^{k \lambda_{n} t}+\omega \cos (\omega \mathrm{t})+\mathrm{k} \lambda_{\mathrm{n}} \sin (\omega \mathrm{t})}{k^{2} \lambda_{n}{ }^{2}+\omega^{2}}\right) .
\end{aligned}
$$

Therein, $\lambda_{n}$ are eigenvalues of a second order homogeneous problem, given by $\lambda_{n}=$ $-(\pi n)^{2}$, and the final solution can be written as:

$$
\psi(x, t)=\sum_{n=1}^{\infty}\left(a_{n}(t, n, \omega) \cdot \sin (\mathrm{n} \pi \mathrm{x})\right) .
$$

It can be seen, that for a fixed mode $n$ of the Fourier series, the amplitude behaves asymptotically like $a_{n} \approx \frac{1}{\omega}$. Hence, the perturbed solution approaches the stationary unperturbed solution for increasing frequencies: $\psi(x, t) \equiv 0$. This explains, what could be seen from the Fig. 2. To estimate further, how perturbations are relaxed 
Fig. 11 Definition of the contour in the complex plane

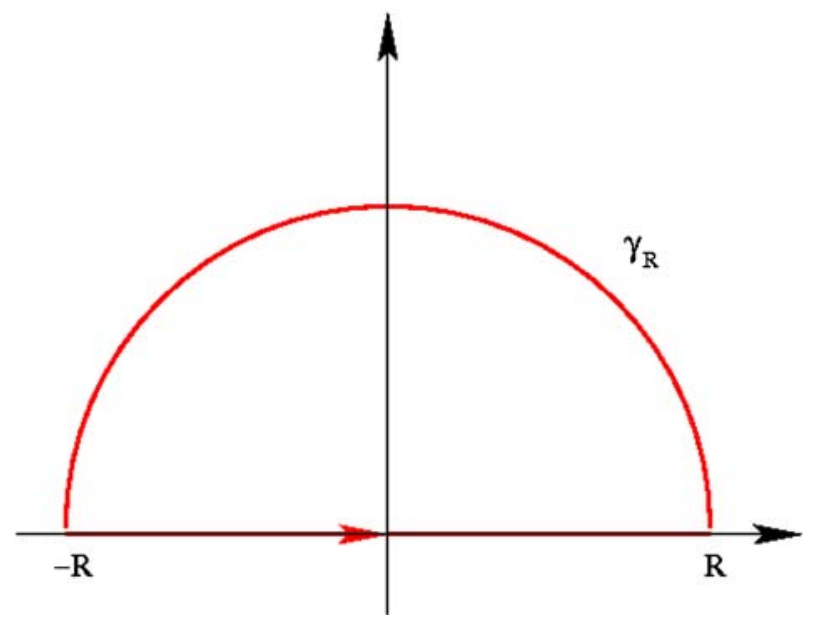

over the spatial interval, we will replace the sum (28) by an integral and apply complex function analysis. First, let us estimate the coefficients $a_{n}$ in (28) for large values of $t$ and a summation index $n>>1$ :

$$
a_{n}=\frac{2 \Lambda k \lambda_{n}}{\pi n}\left(\frac{\omega \cos (\omega t)+k \lambda_{n} \sin (\omega \mathrm{t})}{k^{2} \lambda_{n}^{2}+\omega^{2}}\right) .
$$

Next, we re-write the (28) by using the explicit form of $\lambda_{n}=-(\pi n)^{2}$, with $\mathrm{n}=1, \ldots$ :

$$
\psi(x, t) \approx-\frac{2 \Lambda \omega \cos (\omega \mathrm{t})}{\pi^{3} k} \sum_{n=1}^{\infty} \frac{n \sin (\mathrm{n} \pi \mathrm{x})}{\left(n^{4}+\left(\frac{\omega}{\pi^{2} \cdot k}\right)^{2}\right)}+\frac{2 \Lambda k \sin (\omega \mathrm{t})}{\pi} \sum_{n=1}^{\infty} \frac{n^{3} \sin (\mathrm{n} \pi)}{\left(n^{4}+\left(\frac{\omega}{\pi^{2} \cdot k}\right)^{2}\right)} .
$$

Fig. 12 Sets of the detailed solutions with the estimated function. At the top for $\omega=1$, at the bottom for $\omega=10$; $\Lambda=2$ and $k=0.01$ in both cases
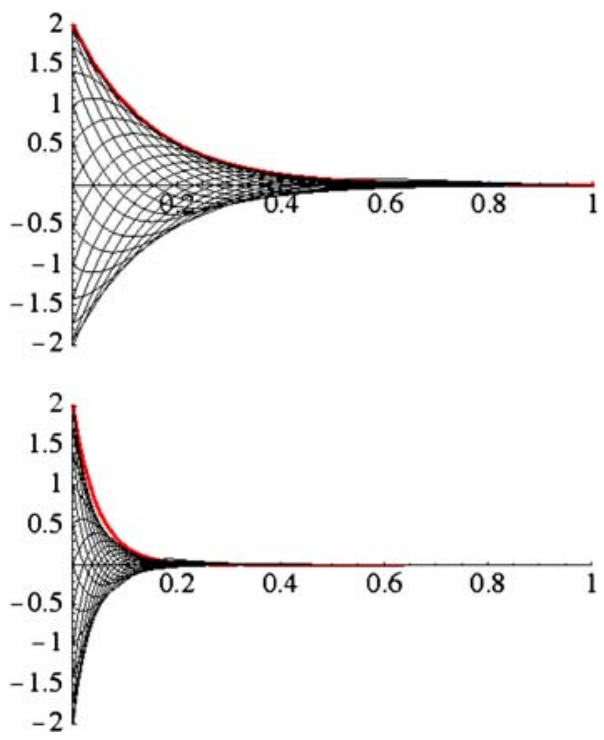
Now, the two left sums in (30) are estimated by integrals:

$$
\begin{aligned}
& S_{1}=\sum_{n=1}^{\infty} \frac{n \sin (\mathrm{n} \pi)}{\left(n^{4}+\left(\frac{\omega}{\pi^{2} \cdot k}\right)^{2}\right)} \approx I_{1}=\int_{0}^{\infty} \frac{z \sin (\mathrm{z} \pi \mathrm{x}) \mathrm{dz}}{\left(z^{4}+a^{2}\right)}, a=\frac{\omega}{\pi^{2} \cdot k} \\
& S_{2}=\sum_{n=1}^{\infty} \frac{n^{3} \sin (\mathrm{n} \pi)}{\left(n^{4}+\left(\frac{\omega}{\pi^{2} \cdot k}\right)^{2}\right)} \approx I_{2}=\int_{0}^{\infty} \frac{z^{3} \sin (z \pi x) d z}{\left(z^{4}+a^{2}\right)} .
\end{aligned}
$$

The converged improper integrals $I_{1}$ and $I_{2}$ can be integrated by a complex contour integration using the Residue Theorem. The integrals are therefore reformulated as complex integrals over the contour shown in Fig. 11 and are solved by residue of the integral function in singular points inside the contour $z=\frac{1}{\sqrt{2}}(1 \pm i)$ :

$$
I_{1}=\frac{\pi}{2 a} e^{-\sqrt{\frac{a}{2}} \pi x} \sin \left(\sqrt{\frac{\mathrm{a}}{2}} \pi \mathrm{x}\right)
$$

and $I_{2}$ can be obtained correspondingly as:

$$
I_{2}=\frac{\pi}{2} e^{-\sqrt{\frac{a}{2}} \pi x} \cos \left(\sqrt{\frac{\mathrm{a}}{2}} \pi \mathrm{x}\right) .
$$

Thus, the behaviour of the solution as a function of the frequency is estimated as:

$$
\begin{aligned}
\psi(x, t, \omega) \approx & -\Lambda \cos (\omega \mathrm{t}) \mathrm{e}^{-\sqrt{\frac{\omega}{2 \mathrm{k}}} \mathrm{x}} \sin \left(\sqrt{\frac{\omega}{2 \mathrm{k}}} \pi \mathrm{x}\right) \\
& +\Lambda k \sin (\omega \mathrm{t}) \mathrm{e}^{-\sqrt{\frac{\omega}{2 \mathrm{k}}} \mathrm{x}} \cos \left(\sqrt{\frac{\omega}{2 \mathrm{k}}} \mathrm{x}\right),
\end{aligned}
$$

which means, that the solution relaxes exponentially according to $\psi(x, \omega) \approx \Lambda e^{-\sqrt{\frac{\omega}{2 k}} x}$ as shown in Fig. 12.

\section{References}

1. Cho, J.H., Lieuwen, T.: Laminar premixed flame response to equivalence ratio oscillations. Combust. Flame 140(1-2), 116-129 (2005)

2. Stahl, G., Warnatz, J.: Numerical investigation of time-dependent properties and extinction of strained methan- and propane-air flamelets. Combust. Flame 85(3-4), 285-299 (1991)

3. Im, H.G., Law, C.K., Kim, J.S., Williams, F.A.: Response of counterflow diffusion flames to oscillating strain rates. Combust. Flame 100(1-2), 21-30 (1995)

4. Fleifil, M., Annaswamy, A.M., Ghonheim, Z.A., Ghoniem, A.F.: Response of a laminar premixed flame to flow oscillations: a kinematic model and thermoacoustic instability results. Combust. Flame 106(4), 487-510 (1996)

5. Lieuwen, T., Zinn, B.T.: The role of equivalence ratio oscillations in driving combustion instabilities in low $\mathrm{NO}_{\mathrm{x}}$ gas turbines. Proc. Combust. Inst. 27, 1809-1816 (1998)

6. Darabiha, N.: Transient behaviour of laminar counterflow hydrogen-air diffusion flames with complex chemistry. Combust. Sci. Technol. 86(1-6), 163-181 (1992)

7. Darabiha, N., Candel, S., Wirth, D.A., Mahan, J.R.: Numerical studis of a pulsing burnerstabilized laminar premixed methan-air flame. Combust. Sci. Technol. 113(1), 35-47 (1996) 
8. Schreel, K.R.A.M., Rook, R., de Goey, L.P.H.: The acoustic response of burner-stabilized premixed flat flames. Proc. Combust. Inst. 29(1), 115-122 (2002)

9. Rook, P., de Goey, L.P.H., Somers, L.M.T., Schreel, K.R.A.M., Parchen, R.: Response of burner-stabilized flat flames to acoustic perturbations. Combust. Theory Model. 6(2), 223-242 (2002)

10. Kornilov, V.N., Schreel, K.R.A.M., de Goey, L.P.H.: Experimental assessment of the acoustic response of laminar premixed Bunsen flames. Proc. Combust. Inst. 31(1), 1239-1246 (2007)

11. Huang, Z., Bechtold, J.K., Matalon, M.: Weakly stretched premixed flames in oscillating flows. Combust. Theory Model. 2(2), 115-133 (1999)

12. Joulin, G., Cambray, P., Jaouen, N.: On the response of a flame ball to oscillating velocity gradients. Combust. Theory Model. 6(1), 53-78 (2002)

13. Tyagi, M., Chakravarthy, S.R., Sujith, R.I.: Unsteady combustion response of a ducted nonpremixed flame and acoustic coupling. Combust. Theory Model. 11(2), 205-226 (2007)

14. Sung, C.J., Law, C.K.: Structural sensitivity, response and extinction of diffusion and premixed flames in oscillating counterflow. Combust. Flame 123(3), 375-388 (2000)

15. Wang, H.Y., Bechtold, J.K., Law, C.K.: Nonlinear oscillations in diffusion flames. Combust. Flame 145(1-2), 376-389 (2006)

16. Metzener, P., Matalon, M.: Diffusive-thermal instabilities of diffusion flames: onset of cells and oscillations. Combust. Theory Model. 10(4), 701-725 (2006)

17. Buckmaster, J., Zhang, Y.: Oscillating edge-flames. Combust. Theory Model. 3(3), 547-565 (1999)

18. Delhaye, S., Somers, L.M.T., van Oijen, J.A., de Goey, L.P.H.: Incorporating unsteady floweffects in flamelet-generated manifolds. Combust. Flame 155, 133-144 (2008)

19. Tomlin, A.S., Turanyi, T., Pilling, M.J.: Mathematical tools for the construction, investigation and reduction of combustion mechanisms. In: Pilling, M.J. (ed.) pp. 293-437. Elsevier, Amsterdam (1997)

20. Smooke, M.D.: Lecture Notes in Physics, p. 384 (1991)

21. Ren, Z., Pope, S.B.: The use of slow manifolds in reactive flows. Combust. Flame 147(4), 243-261 (2006)

22. Maas, U., Pope, S.B.: Simplifying chemical kinetics: intrinsic low-dimensional manifolds in composition space. Combust. Flame 88(3-4), 239-264 (1992)

23. Maas, U., Pope, S.B.: Implementation of simplified chemical kinetics based on intrinsic lowdimensional manifolds. Proc. Combust. Inst. 24, 103-112 (1992)

24. Bykov, V., Maas, U.: The extension of the ILDM concept to reaction-diffusion manifolds. Combust. Theory Model. 11(6), 839-862 (2007)

25. Bykov, V., Maas, U.: Problem adapted reduced models based on Reaction-Diffusion Manifolds (ReDims). Proc. Combust. Inst. 32 (2008, in press)

26. Schmidt, D., Blasenbrey, T., Maas, U.: Instrinsic low-dimensional manifolds of strained and unstrained flames. Combust. Theory Model. 2(2), 135-152 (1998)

27. Warnatz, J., Maas, U., Dibble, R.W.: Combustion. Physical and Chemical Fundamentals, Modeling and Simulation, Experiments, Pollutant Formation. Springer, Berlin (2006)

28. Maas, U., Warnatz, J.: Ignition processes in hydrogen-oxygen mixtures. Combust. Flame 74(1), 53-69 (1988)

29. Davis, M.J.: Low-dimensional manifolds in reaction-diffusion equations. 1. Fundamental aspects. J. Phys. Chem. 110(16), 5235-5256 (2006)

30. Davis, M.J.: Low-Dimensional manifolds in Reaction-diffusion equations. 2. Numerical analysis and method development. J. Phys. Chem. 110(16), 5257-5272 (2006)

31. Bogolyubov, N.N., Mitropolsky, Y.A.: Asymptotic Methods in the Theory of Nonlinear Oscillations. N.Y. Gordon and Breach, Delhi (1961)

32. Fenichel, N.: Geometric singular perturbation theory for ordinary differential equations. J. Differ. Equ. 31(1), 53-98 (1979)

33. Roussel, M.R., Fraser, S.J.: Global analysis of enzyme inhibition kinetics. J. Phys. Chem. 97, 8316-8327 (1993)

34. Roussel, M.R., Fraser, S.J.: Invariant manifold methods for metabolic model reduction. Chaos 11, 196-206 (1993)

35. Singh, S., Rastigejev, Y., Paolucci, S., Powers, J.M.: Viscous detonation in $\mathrm{H}_{2}-\mathrm{O}_{2}-\mathrm{Ar}$ using intrinsic low-dimensional manifolds and wavelet adaptive multilevel representation. Combust. Theory Model. 5, 163-184 (2001)

36. Singh, S., Powers, J.M., Paolucci, S.: On slow manifolds of chemically reactive systems. J. Chem. Phys. 117(4), 1482-1496 (2002) 
37. Hadjinicolaou, M., Goussis, D.A.: Asymptotic solution of tiff PDEs with the CSP method: the reaction diffusion equation. SIAM J. Sci. Comput. 20, 781-910 (1999)

38. Valorani, M., Goussis, D.A.: Explicit time-scale splitting algorithm for stiff problems: autoignition of gaseous mixtures behind a steady stock. J. Comput. Phys. 169(1), 44-79 (2001)

39. Gicquel, O., Darabiha, N., Thevenin, D.: Laminar premixed hydrogen/air counterflow flame simulations using flame prolongation of ILDM with differential diffusion. Proc. Combust. Inst. 28, 1901-1908 (2000)

40. Bykov, V., Maas, U.: Extension of the ILDM method to the domain of slow chemistry. Proc. Combust. Inst. 31(1), 465-472 (2007)

41. Golub, G.H., van Loan, C.F.: Matrix Computations. The John Hopkins University Press, Baltimore (1996)

42. Maas, U.: Efficient calculation of intrinsic low-dimensional manifolds for the simplification of chemical kinetics. Comput. Vis. Sci. 1(2), 69-82 (1998)

43. König, K., Maas, U.: Sensitivity of instrinsic low-dimensional manifolds with respect to kinetic data. Proc. Combust. Inst. 30(1), 1317-1323 (2005) 